EXPERIMENTAI NEUROLOGY 10, 296-304 (1964)

\title{
Subcortical Lesions and Maze Retention in the Rat
}

\author{
M. E. Olds and David Hogberg \\ Department of Psychology, The University of Michigan, Ann Arbor, Michigan
}

Received April 9, 1964

\begin{abstract}
With intracranial electric shock in the posterior hypothalamus as the source of positive reinforcement, eleven rats were trained to run and "count" in an operant version of the Lashley III maze. In seven animals, bilateral lesions were made in anterior medial forebrain bundle regions; in four animals bilateral lesions were made in anterior thalamic and caudate regions. In earlier experiments anterior medial forcbrain bundle lesions had failed to abolish a simple Skinner box behavior rewarded by electric stimulation in the posterior hypothalamus. The possibility was raised that if the response were more complex, lesions in anterior medial forebrain bundle, severing connections between paleocortex and hypothalamus, might cause deficits. The data confirmed this supposition. Four weeks after lesions in anterior medial forebrain bundle, there was almost total deficit in maze and counting behavior. Thirteen weeks after lesions some recovery had occurred, but counting still showed large deficits. Independent Skinner box tests showed that most of these rats were still reinforced by the hypothalamic stimulation. Lesions in thalamic and caudate areas caused minor and temporary deficits. The conclusion was drawn that paleocortical-hypothalamic connections are important when hypothalamic stimulation is used to reinforce behaviors which are complex in character in the sense of drawing upon memory or integrative factors.
\end{abstract}

\section{Introduction}

Current indications are that the cortical association area proximal to a primary sensory area may be involved in learning relevant to that area, and that more general association areas may be related to aspects of learning which are difficult to define $(4,8)$. Nevertheless, Lashley's $(6)$ concepts of equipotentiality and mass action are still relevant to the broader problem of learning and memory; and there is still some reason to suspect that the main mechanisms involved in learning are either not located in the cortex or not localized at all.

Proceeding on the assumption that the learning mechanisms might have a subcortical locus, several investigators have recently applied discrete electrolytic lesions in various subcortical structures $(14,16)$. Subcortical lesions proved surprisingly effective in the study of large defects with 
minimal tissue destruction, but what pattern they indicate is a question still to be resolved.

Subcortical areas are involved in all major aspects of function. There are primary sensory, attentional, visceral, autonomic, and directional or motivational systems. Many of these appear to be heavily overlapped. Disruption of sensory, attentional, or motivational functions might be expected to yield "learning" defects even though the primary mechanisms of learning might not be involved.

Although it is not crucially significant to the locus of engram formation, it is nonetheless interesting to find whether lesions in sensory, attentional, or motivational systems have marked effects on learning.

The work of Rosvold and Delgado (12), Thomas and Otis (14), and Thompson and Hawkins (16) have focused attention on caudate and interpeduncular nuclei and on various other aspects of nonspecific systems of the thalamus, hypothalamus, and midbrain. Studies aimed at creating a temporary nexus of disorganization by maintaining a suprathreshold field of electrical stimulation seem to point to these same systems. In particular, electric stimulation in nonspecific thalamic structures, in attentional regions of the tegmentum, in the caudate nucleus, and in motivational regions of the hypothalamus has been shown to disrupt associational processes $(2,7$, $11-13,15$ ).

Work on the hypothalamus has been interpreted as if the stimulation merely provoked a motivational effect which interfered with normal performance and not with memory. Such an interpretation would be weakened if lesions produced effects similar to those of electric stimulation in the same areas, as Rosvold and Delgado (12) showed in the caudate nucleus. In experiments by Thompson and associates $(17,18)$, lesions in interpeduncular and nearby areas served to disrupt $\mathrm{T}$-maze learning. Because stimulation in or around these areas has been shown to have positive reinforcing effects (10) and because the reinforcement of brain stimulation in other experiments (11) has disrupted associational processes, it appears that lesions may be effective against learning in the same motive systems where stimulation disrupts learning. To check this possibility, another part of the positive reinforcing system, namely, the other end of the lateral hypothalamic neural tube--where the medial forebrain bundle (MFB) passes from the hypothalamus into the telencephalon-was tested for the effects of lesions on learning.

Lesions in this "motivational nexus" were compared with those in a more associational-sensory point, the thalamus. As the MFB lesions were 
placed where stimulation had both memory-disrupting and motive effects and as this lesion site was at the other end of the hypothalamic tube from the interpeduncular area (where lesions are known to interfere with memory), any memory defects caused by the MFB lesions might be taken to indicate possible coexistence of reinforcing and mnemonic functions in subcortical structures. Profound disruption of both maze and cognitive performances was in fact produced by these lesions, which suggests that there may be some close relation between the motivational properties of the rostral subcortical structures and the formation of effective memory traces. The outcome certainly does not indicate that memory traces are formed in these structures, but it does suggest the possibility of a closer relation between these anatomical areas and learning than one would expect from knowledge of their motivational and vegetative functions alone.

\section{Methods}

Eleven rats with rewarding electrodes implanted in the MFB at the level of the posterior hypothalamus were trained to run and "count" in an operant version of the Lashley III maze. Pedals were placed at the start and goal positions in the maze. The goal pedal yielded one electric shock to the brain (ESB) - a reward stimulus-for each of three responses, after which an automatic programmer deactivated the goal pedal and activated the start pedal. The rat could then retrace the maze and receive another three ESB rewards from the start pedal, following which the programmer reversed the lever activation again. The animals learned not only to shuttle through the maze for the central nervous system (CNS) rewards but also to count, i.e., to eliminate wasted responses. A pedal response was wasted when a rat pressed the activated pedal more than three times in succession; a run was wasted when a rat pressed the pedal only once or twice before running to the other lever which, accordingly, was still inactive. When a rat wasted a run it did not receive an ESB reward again until it returned to the neglected lever. There was no special punishment for errors in counting, although it could be said that overcounting was "punished" by the effort expended in the wasted pedal pressing, which seemed little enough; undercounting, by the same token, was punished by the extra effort wasted in the maze run. Most animals, after 1 or 2 months of training $(2 \mathrm{hr}$, twice a week), counted accurately more than 80 per cent of the time. All the rats used in the present experiment met this criterion. 
In order to speed the acquisition of maze and counting skill, the rats were constrained by barriers to a short path between pedals when they were first placed in the maze. After the animals began to develop good shuttling and counting behavior, the barriers were gradually positioned to form the Lashley III maze. To permit suspension of stimulating leads, the top of the maze was left open; sides $25.5 \mathrm{~cm}$ high prevented escape. Each ESB was a $0.5-\mathrm{sec}$ train of sine wave stimuli at $60 \mathrm{cycle} / \mathrm{sec}$, with current set at $70 \mu \mathrm{amp}$ rms. With this reinforcement all rats used in the maze tests had demonstrated high Skinner box rates (more than 600 responses per 8 -min test).

In the counting maze an adequate measure, for the purposes of the present experiment, was the length of time it took an animal to reach sixty successful lever responses, i.c., twenty groups of three rewards. When the animal was counting well, this was a matter of twenty maze runs, ten in each direction, and of sixty responses. When it was counting poorly, this was a matter of more responses, or more runs or both; and when it was making errors, each maze run took longer. Thus the time of sixty successful responses represents an integral score for evaluating simultaneously the various aspects of maze performance. Because the defects produced by the anterior bilateral subcortical lesions were large and apparent in all aspects of the cognitive performance, the time measure is used to show them. All scores in Table 1, therefore, are expressed in terms of the time it took an animal to achieve a criterion of sixty reinforced responses on a given day, or an inversion of this score in terms of maze runs per hour. Time scores ranged from $3 \mathrm{~min}$ to more than $2 \mathrm{hr}$. A 3-min time score indicated 20 runs in $3 \mathrm{~min}$ or $400 \mathrm{run} / \mathrm{hr}$; by the same conversion, a 2-hr time score indicated $10 \mathrm{run} / \mathrm{hr}$. In addition, total number of responses and percentage of correct responses were graphed as a function of weeks.

Bar-pressing rate for the same electric-shock reinforcement in a simple Skinner box was simultaneously used to measure general health, activity, and motivation. Self-stimulation tests were administered 4 weeks before and weekly after lesions. Large deficits in maze and counting performance unaccompanied by similarly large deficits in self-stimulation were taken to indicate deficits in cognitive performance.

Animals were trained until they achieved the criterion of sixty reinforcements in $5 \mathrm{~min}$ or less in ten consecutive tests. Bilateral lesions were then made stereotaxically under Nembutal anesthesia after the Kricg (5) 
method. Direct current of $2 \mu \mathrm{mp}$ was applied for $15 \mathrm{sec}$ by a wire with an uninsulated $0.5-\mathrm{mm}$ tip. Following a 4-day period after lesions, tests were resumed on the 2-day-per-week schedule. Results for all the rats are

TABLE 1

Cognitive and Motivational Performance Score Before and After Electrolytic Lesions in MFB and Medial Geniculate Body (Control) a

\begin{tabular}{|c|c|c|c|c|c|c|}
\hline \multirow{2}{*}{$\begin{array}{l}\text { Rat } \\
\text { No. }\end{array}$} & \multicolumn{2}{|c|}{ Cognitive $^{b}$} & \multirow{2}{*}{$\begin{array}{c}\mathbf{A} / \mathbf{B} \\
\%\end{array}$} & \multicolumn{2}{|c|}{ Motivational $^{\circ}$} & \multirow{2}{*}{$\begin{array}{c}\mathrm{C} / \mathrm{D} \\
\%\end{array}$} \\
\hline & A & B & & $\mathrm{C}$ & D & \\
\hline \multicolumn{7}{|c|}{ MFB lesions } \\
\hline 4808 & 420 & 18 & 4 & 750 & 975 & 130 \\
\hline 5653 & 150 & 8 & 5 & 690 & 250 & 36 \\
\hline 4811 & 240 & 10 & 4 & 789 & 450 & 57 \\
\hline 4560 & 420 & 10 & 2 & 736 & 300 & 41 \\
\hline 4558 & 300 & 10 & 3 & 518 & 200 & 39 \\
\hline 6575 & 240 & 10 & 4 & 500 & 249 & 50 \\
\hline 6475 & 170 & 36 & 21 & 458 & 80 & 17 \\
\hline Mean & & & 6 & & & 54 \\
\hline \multicolumn{7}{|c|}{ Other lesions } \\
\hline 5764 & 300 & 300 & 100 & 565 & 250 & 44 \\
\hline 4711 & 300 & 150 & 50 & 550 & 450 & 82 \\
\hline 6578 & 240 & 220 & 92 & 650 & 456 & 70 \\
\hline 6477 & 180 & 120 & 67 & 714 & 719 & 100 \\
\hline Mean & & & 79 & & & 74 \\
\hline
\end{tabular}

a All postlesion scores were taken on the seventh test during the fourth week after surgery.

${ }^{b}$ Cognitive performance score in runs per hour. A, before lesions; B, after lesions.

$c$ Motivational performance score in self-stimulation responses per 8-min test. C, before lesions; D, after lesions.

presented for the seventh postlesion test (performed during the fourth postoperative week) and for the test performed just before lesions (Table 1).

Approximately 3 months after lesions, the rats were killed and their brains prepared histologically to permit localization of the lesions and electrodes.

\section{Results}

Histological examination showed that ten rats had self-stimulating electrodes in the posterior hypothalamus at the level of the mamillary complex. In each animal the rewarding properties of electric shock to that part of 
the brain were very strong as measured by the rates of 500 to 700 autostimulation responses per 8-min session when stimulation at a level of 50 uamp rms was used. In seven rats the bilateral anterior subcortical lesions were localized in the very anterior hypothalamus at the level of nucleus accumbens, the tuberculum olfactorum, and the anterior area of the amygdala. These lesions were at the same depth as the stimulating macroelectrodes but impinging on the very anterior end of the tube formed by the MFB; the stimulating, rewarding electrodes were at the posterior end of the tube. In three rats the lesions were also placed anterior to the stimulating electrodes, approximately at the same level on the longitudinal axis as the aforementioned lesions in the seven rats; however, in these cases the lesions were not at the same depth as the electrodes but were higher up at the level of the ventral nucleus and the reticular nucleus of the thalamus.

In one rat the stimulating electrodes were in the anterior MFB and the lesions were posterior, in the geniculate body, impinging on the midbrain tegmentum. This animal was added to the three rats with lesions in the thalamus to be used as control subjects.

In all seven rats with bilateral lesions in the anterior MFB overlapping adjoining nuclei in the hypothalamus and amygdala, the percentage of correct counting responses and the number of maze runs per hour decreased sharply after the lesions and in six cases did not return to even the 50 per cent level 15 weeks after the lesions, despite biweekly training sessions of $2 \mathrm{hr}$ each day. The self-stimulation tests given to rats after making lesions showed that the animals were still motivated-the rates of pedal-pressing for ESB varied between 80 and 975 during an 8-min period, and averaged 54 per cent of prelesion levels. In only one rat did the rewarding electrodes lose their rewarding properties after lesions (Table 1).

In contrast, the four rats that had bilateral lesions in the thalamus did not show long-lasting decrements in counting behavior although there was some reduction in the self-stimulation rates of the rewarding electrodes after lesions (Table 1).

To summarize: In all animals with bilateral electrolytic lesions placed anteriorly in approximately the same anatomical structure that had a posteriorly placed rewarding electric probe, the cognitive performance was severely affected despite the survival of some motivational properties in the stimulating electrodes. In animals with lesions in thalamic structures cogni- 
tive performance was not similarly impaired even though there were some motivational delicits.

\section{Discussion}

Motivation of Behavior Versus Motivation of "Thought" Processes. The marked debilitating effect of anterior lesions on maze and counting behavior driven by posterior stimulation in the MFB was in sharp contrast with the relative absence of effect of rostral lesions on the simpler selfstimulation behavior driven by the same stimulation. Telencephalic lesions in the amygdala and septal area failed to counter self-stimulation by electrodes in the ventrolateral midbrain $(19,20)$, and cingulate lesions failed to disrupt hypothalamic self-stimulation (1). Moreover, anterior lesions in the MFB had only moderate attenuating effect when tested in relation to self stimulation in the posterior MFB; in some experiments posterior lesions often completely abnegated anterior self-stimulation (9). The repeated failure to impair self-stimulation behavior by damaging the connections of the self-stimulation point to the telencephalon was puzzling in view of the widespread belief that operant or voluntary behavior must be a "cortical" function.

The present data suggest that very great deficits are made by lesions between the self-stimulation electrodes and the telencephalon when more "intellectual" behaviors are to be motivated by the ESB incentive. It therefore seems attractive to guess that the MFB acts downward to motivate "mere behavior" and upward to motivate "thought processes," in a fashion similar to that suggested in the Cannon and Bard reinterpretation of the James-Lange theory of emotions.

The Problem of Extinction in Relation to Counting and Maze Running. Howarth and Deutsch (3) found that in certain cases self-stimulation extinction occurred in $10 \mathrm{sec}$, irrespective of extinction responses during the interval. They spoke of extinction as a function of time alone. Wetzel (21) also showed that some rats only seemed positively motivated for a few minutes after the last stimulation. In these animals priming stimulation was needed to cause runway performances for ESB incentives. It was also clear from Wetzel's work and from many unpublished observations in our laboratory that some self-stimulation points do not yield this "quick extinction." The difference is not clear but we assume it is based on the finer details of electrode placement.

In the present experiment we also found that some electrodes yielded positive reinforcement of such a nature that it easily promoted and sus- 
tained maze and counting behavior. Other electrodes did not. Again we do not know the source of the difference, but we assumed it was based on microdifferences in the locus of electrode tips. We did not use the nonmaze performers in these experiments.

It is appealing to suppose that the electrodes which did not promote learning were the Deutsch "quick extinction" probes because the animal, quickly forgetting the incentive, would not learn to traverse the maze or to count. If this were so, then it also appears from the present experiment that the maze learners might be transformed into the Howarth and Deutsch "quick forgetters" by means of the anterior lesions. That this interpretation cannot be entirely true was indicated by the extremely persevering extinction behavior exhibited by some of the rats with brain injuries. While unable to count or find their way through the maze, they often repeatedly pressed the noneffective lever after repeated failures to traverse the maze. If we suppose that connections via the anterior area are important both to sustained maze performance and to the negative reinforcement consequent on frustration, then we might also suppose that anterior lesions rendered rats likely to "forget" more quickly, but less negatively reinforced during persevering extinction behavior.

\section{References}

1. Fonberg, E., and J. M. R. Delgado. 1961. Inhibitory effects of amygdala on food intake and conditioning. Federation Proc. 20: 335.

2. Glickman, S. E. 1958. Deficits in avoidance learning produced by stimulation of the ascending reticular formation. Can. J. Psychol. 12: 97-102.

3. Howarth, C. I., and J. A. Deutsch. 1962. Drive Decay: The cause of fast "extinction" of habits learned for brain stimulation. Science 137: 35-36.

4. Konorski, J. 1961. The physiological approach to the problem of recent memory, pp. 115-132. In "Brain Mechanisms and Learning," A. Fessard, et al. [ed.]. Blackwell, Oxford.

5. KRIEG, W. J. S. 1946. Accurate placement of minute lesions in the brain of the albino rat. Quart. Bull. Northwestern Univ. Med. School 20: 199-208.

6. LaShley, K. S. 1929. "Brain Mechanisms and Intelligence." Univ. of Chicago Press, Chicago, Illinois.

7. Maнut, H. 1957. Effects of subcortical electrical stimulation on learning in the rat. Am. Psychologist 12: 466.

8. Miskin, M., and K. H. Pribram. 1956. Analysis of the effects of frontal lesions in monkeys. II. Variations of delayed response. J. Comp. Physiol. Psychol. 49: $36-40$.

9. Olds, J., and M. E. Olds. 1964. Mechanisms of Voluntary Behavior. In "The Role of Pleasure in Behavior," R. G. Heath [ed.]. Harper (Hoeber) New York. In press. 
10. Olds, J., R. P. Travis, and R. C. Schwing. 1960. Topographic organization of hypothalamic self-stimulation functions. J. Comp. Physial. Psychol. 53: 23-32.

11. OtDs, M. E., and J. OtDs, 1961. Emotional and associative mechanisms in rat brain. J. Comp. Physiol. Psychol. 54: 120-126.

12. Rosvold, H. E., and J.M.R. Delcado. 1956. The effect on delayed alternation test performance of stimulating or destroying electrically structures within the frontal lobes of the monkey's brain. J. Comp. Physiol. Psychol. 49: 365-372.

13. Stein, L., and E. Hearsir. 1958. Inhibitory effect of positively reinforcing brain stimulation on learning. Am. Psychologist 13: 408.

14. Thомas, G. J., and L. S. Otis. 1958. Effects of rhinencephalic lesions on maze learning in rats. J. Comp. Physiol. Psychol. 51: 161-166.

15. THOMPson, R. 1958. The effect of intracranial stimulation on memory in cats. J. Comp. Physiol. Psychol. 51: 421-426.

16. Thompson, R. 1961. The interpeduncular nucleus and retention in albino rats. J. Comp. Physiol. Psychol. 54: 329-333.

17. Thompson, R., and W. F. Hawkins. 1961. Memory unaffected by mammillary body lesions in the rat. Exptl. Neurol. 3: 189-196.

18. Thompson, R., and L. C. Massopust. 1960. The effect of subcortical lesions on retention of a brightness discrimination in rats. J. Comp. Physiol. Psychol. 53: 488-496.

19. WARD, H. P. 1960. Basal tegmental self-stimulation after septal ablation in rats. A.M.A. Arch. Neurol. 3: 158-162.

20. WARD, H. P. 1961. Tegmental self-stimulation after amygdaloid ablation. A.M.A. Arch. Neurol. 4: 657-659.

21. Wetzes, M. C. 1963. Self-stimulation aftereffects and runway performance in the rat. J. Comp. Physiol. Psychol. 56: 673-678. 